\title{
Association Between Gestational Bell's Palsy and Preeclampsia and Other Pregnancy-Related Metabolic Disorders: A Retrospective Comparative Case Study
}

\author{
Sumonthip Leelawai \\ Prince of Songkla University \\ Chitkasaem Suwanrath \\ Prince of Songkla University \\ Nannapat Pruphetkaew \\ Prince of Songkla University \\ Pensri Chongphattararot \\ Prince of Songkla University \\ Pornchai Sathirapanya ( $\sim$ sporncha@medicine.psu.ac.th ) \\ Prince of Songkla University
}

\section{Research Article}

Keywords: Bell's palsy, eclampsia, preeclampsia, pregnancy, hypertension, diabetes

Posted Date: January 8th, 2021

DOI: https://doi.org/10.21203/rs.3.rs-138913/v1

License: (c) (1) This work is licensed under a Creative Commons Attribution 4.0 International License.

Read Full License 


\section{Abstract}

\section{Background}

The associations between gestational Bell's palsy (GBP) and preeclampsia (PE) and other pregnancyrelated metabolic disorders such as gestational hypertension (GHT) and gestational diabetes mellitus (GDM) remain unclear. We aimed to evaluate these associations and to compare the neonatal outcomes between the newborns of pregnant women with and without GBP.

\section{Methods}

This was a retrospective 1:5 comparative case study between pregnant women with and without GBP. GBP cases in Songklanagarind Hospital from 2006 to 2016 were enrolled. The exact maternal age and order of gravidity at the on set of GBP were matched. The associations between GBP and PE, GHT and GDM were evaluated comparatively. Also, the outcomes of newborns from the two pregnant women groups were compared and analyzed by univariable analysis $(p<0.05)$.

Results

Eight GBP cases out of 8,756 pregnant women were enrolled. Six of the GBP cases were first or second gravidity pregnancies. Five cases developed GBP during the third trimester. Except for significantly higher median systolic and diastolic blood pressures (SBP and DBP) in the GBP cases, we found no significant associations between GBP and PE, GHT or GDM. Additionally, the neonates of the GBP women had significantly lower mean birth weight.

\section{Conclusions}

We found that GBP had no associations with PE, GHT or GDM. However, significantly lower birth weight was found in the newborns of the GBP women. The higher median SBP and DBP found in the GBP women may imply that a common pathogenesis of microvasculopathy between GBP and late pregnancyrelated complications exists.

\section{Background}

The pathogenesis of Bell's palsy (BP) has not yet been clearly explained. The two accepted proposals for this pathogenesis are reactivation of the herpes simplex virus (HSV) which inhabits the geniculate ganglions of the facial nerves as a latent infection, and ischemic facial neuropathy associated with diabetes and other metabolic disorders similar to diabetic oculomotor cranial neuropathy

[1]. BP occurring during pregnancy, called "gestational Bell's palsy (GBP)" in this study, commonly takes place during the late third trimester or the immediate postpartum period which is the same periods as preeclampsia (PE) or eclampsia (EC) usually happen. Due to the same period of appearance of GBP and $\mathrm{PE}$ or EC, it is believed that they may share a common pathogenesis [2], and GBP is proposed as a risk for 
PE or EC as well [3-6]. This presumption is based on only a few case reports, case series and unmatchedcase comparison study. Therefore, this study aimed to investigate whether there are significant associations between GBP and PE, EC and other pregnancy-related metabolic disorders, i.e. gestational hypertension (GHT) and gestational diabetes (GDM), in a matched- case study design. The neonatal outcomes of newborns born from GBP (GBP+) and non-GBP (GBP-) pregnant women were comparatively analyzed for significant differences in the neonatal development scales as well.

\section{Methods}

\section{Study participants, design and setting}

This was a retrospective 1:5 comparative case study matching the exact maternal age and the order of gravidity at the onset of GBP between GBP+ and GBP- pregnant women. The newborns of GBP+ and GBP- pregnant women were matched corresponding to their mothers, and their neonatal characteristics also comparatively analyzed for significant differences. We retrospectively enrolled the study cases from the pregnant women who completed their pregnancies and delivered alive babies between January 2006 and December 2016 in Songklanagarind Hospital, a 900-bed referral and medical teaching hospital affiliated with Prince of Songkla University in southern Thailand. We defined GBP+ cases as pregnant women who acquired BP during their pregnancies and until 6 weeks postpartum. We used the Random.org website to randomly select GBP- pregnant women as controls from the hospital information recording system. All the enrolled pregnant women had regular follow-ups and complete obstetric history records in our center. We excluded pregnant women with a known history of essential hypertension or overt diabetes mellitus, or both, before the index pregnancy.

\section{Terms and Definitions}

a) Bell's palsy is defined by an acute unilateral peripheral facial paralysis without an identifiable cause.

b) Gestational hypertension (GHT) is an elevation of systolic blood pressure (SBP) $\geq 140 \mathrm{mmHg}$ or diastolic blood pressure (DBP) $\geq 90 \mathrm{mmHg}$ measured after 20 weeks of gestation without proteinuria, in a previously normotensive woman, and the elevated SBP and DBP normalize by 12 weeks postpartum.

c) Gestational diabetes mellitus (GDM) we followed the diagnostic criteria of the American College of Obstetricians and Gynecologists as follows: if the one-hour plasma glucose level of a 50-gram oral glucose challenge test (50-g GCT) is $\geq 140 \mathrm{mg} / \mathrm{dL}$, then a three-hour, 100-gram oral glucose tolerance test (100-g OGTT) is further done. A diagnosis of GDM is made when two or more of the plasma glucose levels meet the pre-defined levels. The pre-defined plasma glucose levels of 100-g OGTT are as follows:

a) fasting plasma glucose $\geq 95 \mathrm{mg} / \mathrm{dL}$, b) $1^{\text {st }}$-hour plasma glucose $\geq 180 \mathrm{mg} / \mathrm{dL}$, c) $2^{\text {nd }}$-hour plasma glucose $\geq 155 \mathrm{mg} / \mathrm{dL}$, or d) $3^{\text {rd }}$-hour plasma glucose $\geq 140 \mathrm{mg} / \mathrm{dL}$. Additionally, overt diabetes mellitus in pregnancy is defined by a fasting plasma glucose level $>126 \mathrm{mg} / \mathrm{dl}$, or 2-hour plasma glucose $>200$ $\mathrm{mg} / \mathrm{dL}$ following a 75 -gram oral glucose loading test, or random plasma glucose $>200 \mathrm{mg} / \mathrm{dL}$ in the presence of diabetic symptoms. 
d) Preeclampsia (PE) is a pregnancy with $\mathrm{SBP} \geq 140 \mathrm{mmHg}$ or DBP $\geq 90 \mathrm{mmHg}$ plus at least one of the following features: a) $1+$ proteinuria on a urine dipstick, b) 24-hour urine protein $>300 \mathrm{mg}$, or c) a urine protein-to-creatinine ratio $\geq 0.3$.

e) Eclampsia (EC) is defined as the clinical features of PE plus seizures that are not attributable to other causes.

\section{Data collection}

GBP+ and GBP- patients' demographic data, parameters of metabolic disorders, current obstetric profiles, and gestational age at the onset of GBP were recorded. The presence of PE, EC, GHT, and GDM were carefully explored from the medical records and identified based on the diagnostic criteria mentioned before. We specifically recorded the maternal weight, height, body mass index (BMI), SBP and DBP of $\mathrm{GBP}+$ cases at the time of GBP occurrence, and those of GBP- cases at a comparable gestational age $( \pm 2$ weeks). We also collected characteristics of the newborns of the GBP+ and GBP-women including birth weight, body length, Apgar scores and routes of delivery for comparative analysis.

\section{Statistical analysis}

Descriptive statistics were used to compare the baseline characteristics between the GBP+ and GBPwomen. Wilcoxon Rank Sum test, t-test and Fisher's exact test were used for demonstrating significant differences $(p<0.05)$ in the characteristics between the two groups and corresponding newborns. Associations between GBP and PE, EC, GHT and GDM were analyzed by univariable analysis $(p<0.05)$.

\section{Ethical considerations}

The study protocol was approved by the Ethics Committee of the Faculty of Medicine, Prince of Songkla University (EC Approval Registration No. 60-132-14-4) We strictly followed the regulations in the 1964 Declaration of Helsinki and its following amendments. We also followed the current good clinical practice guidelines in doing this research. We completely anonymized the identifiable personal information of the study cases.

\section{Results}

There were 8 out of $8,756(0.09 \%)$ pregnant women who met the inclusion criteria of GBP+ during the study period. Forty control cases (GBP-) were randomly selected using the mentioned website. The median (IQR) maternal age at the onset of BP was $31.5(26.8,36.2)$ years. GBP occurred during the first (3 cases), second ( 3 cases), and fourth and fifth (1 case each) gestations. The onset of GBP was in the late second trimester in 2 cases, the third trimester in 5 cases, and postpartum in 1 case. Among the GBP+ pregnant women, we identified only one case with PE, and one other case with both GDM and GHT. No cases associated with EC were found. 
There were no significant differences in maternal weight, height and BMI between cases and controls groups. We found only significantly higher median (IQR) SBP and DBP in the GBP+ group by univariable analysis. However, both the elevated blood pressures were not over the accepted limits to meet the diagnostic criteria of GHT. The median (IQR) of 50-g GCT in the GBP+ group was non-significantly higher than in the GBP-group. No significant associations between GBP and the development of GHT, GDM, PE and EC were found (Table 1).

In comparisons of the neonatal outcomes, except for a significantly lower average birth weight in the newborns of the GBP+ women, there were no significant differences in body length, APGAR scores at 1 and 5 minutes, or route of delivery (Table 2).

Because of the very small number of GBP+ cases enrolled in our study, we could not proceed to do multivariable analysis of the study data.

\section{Discussion}

Contrary to the previous studies, we found no associations between GBP and PE or EC, or metabolic complications during pregnancy. The only significant finding in our study was higher SBP and DBP in $\mathrm{GBP}+$ pregnant women, but these elevated blood pressures did not fulfil the diagnostic criteria for GHT. As most of the previous studies reporting a higher risk of PE among the pregnant women with GBP were based on merely a few cases reports or small case series, the reliability of their conclusions is quite doubtful. To date, there have been few large comparative case studies which conclude that GBP was "probably" associated with GHT, $\mathrm{PE}$, and other pregnancy-related adverse events. For example, one study found that GBP women experienced a six-fold higher incidence of PE and a 1.5-fold higher incidence of GHT compared to the expected rates in the general female population [3]. Another study also found significantly higher rates of severe PE and cesarean delivery but the neonatal outcomes were still favorable [4]. It is noteworthy that although both of these studies included a larger number of GBP+ cases than earlier studies, the former study compared the real incidences of PE and GHT calculated with the expected incidences among the general female population from a nationwide survey reported in another study, while the latter was not a comparable matched-case study between GBP+ and GBP-pregnant cases. Therefore, we conclude that a significant association between GBP and PE or GHT has not been confirmed based on the available data.

In addition, Vabrec, et al [7]. also argued against the higher incidences of BP during pregnancy described in some studies. They suggested that the exaggeration in enrollment of controls plus active recruitment of pregnant women with GBP in those studies were able to explain the overestimations of the incidence of GBP among pregnant women. For these reasons, the reported incidence of GBP of 45:100,000 "live births" compared with 17:100,000 "women of child bearing age" used as controls in a study by Hilsinger, et al. [8] was arguable. Based on their argument on this issue, the previous agreement that the incidence of BP during pregnancy is higher than in the general female population seems unreasonable. 
Although the higher incidence of BP during pregnancy is controversial, some case reports and small case series associated the occurrence of BP during pregnancy with PE, EC, GHT and GDM. How these disorders are related has been a question waiting for an explanation. Considering the pathogeneses of these pregnancy-related disorders would be helpful. For BP, the definite pathogenesis has not been clearly elucidated. The main theory currently is that BP is caused by reactivation of a herpes simplex virus (HSV) that has stayed inactively in the geniculate ganglions of the facial nerves. The other pathogeneses proposals are 1) ischemic facial neuropathy complicated with metabolic disorders such as long-standing systemic hypertension, glucose intolerance, or diabetes mellitus, 2) altered host immunity predisposing the patients to the reactivation of an HSV, or other infections, and 3) immunologically inflammatory demyelination of the facial nerve, like a variant form of Guillain-Barre syndrome.

According to the currently accepted pathogenic mechanism for PE, the inadequate intrusion of placental trophoblasts into the uterine muscles, combined with insufficient remodeling of the spiral arteries of the uterus during placental implantation cause the disorder [9]. The under development of the spiral arteries results in placental ischemia that triggers an alteration in the proportions of the T lymphocyte subpopulations, i.e. increased numbers of CD4+ and helper-17 cells but decreased regulatory $T$ cells. Following these disproportionate number of $\mathrm{T}$ lymphocyte subpopulations, $\mathrm{B}$ lymphocytes are triggered to release autoantibodies to activate angiotensin II type I receptors. Finally, increased vascular sensitivity to angiotensin develops causing decreased renal blood flow and glomerular filtration rate, and eventually pregnancy-related systemic HT develops [10]. However, based on the available information, how GBP relates to PE is still unsettled, whether a) they share a common pathogenesis of increased vascular sensitivity to angiotensin that causes facial nerve ischemia, or b) GBP is a complication of the systemic $\mathrm{HT}$ associated with $\mathrm{PE}$, or $\mathrm{c}$ ) the changes in host immunity during pregnancy give rise to HSV reactivation or facial nerve demyelination resulting in BP. Our comments on this issue based on the available data are:

1) Reactivation of HSV or other human herpes viruses $(\mathrm{HHV})$ is the more reasonable explanation. Because of the lowered host-defense immunity against infections during pregnancy, pregnant women are predisposed to any infection, including HHV. One study reported a cytomegalovirus infection developed four days before delivery in a pregnant woman [11]. Another study suggested that physiological changes and low host immunity against infections during the third trimester of pregnancy and early puerperium contributed to increased risk of acquiring HHV reactivation and other infections [4].

2) GBP is a complication of systemic HT that is associated with PE. This pathogenic process induces microangiopathy of the vasa nervorum which provides blood supply to the facial nerves. However, as the rising of blood pressures during PE are a transient physiological change that usually resolves shortly after childbirth, we think that the duration of high blood pressure in PE may not be long enough to cause such microvascular complications. Thence, this suggested pathogenesis should be further investigated.

3) Concurrent microvasculitis of the vasa nervorum of the facial nerves with the placental vessels are caused by immune-mediated insults. This pathogenesis also remains inconclusive and requires further study. 
We believe that the lowered host-defense immunity against several infections during pregnancy is a major predisposing factor which hastens the reactivation of inactive HHV and frees them from the geniculate ganglions to cause facial neuropathy. Additionally, physiological changes during near-term pregnancies such as changes in the levels of estrogen, progesterone and corticosteroids possibly cocontribute to the occurrence of facial neuropathy by further impairing the host-defense immunity against the infections [4].

The significantly lower mean birth weight in the newborns of GBP+ women was the only abnormal neonatal development parameter found in our study. This finding possibly resulted from placental vascular insufficiency in the fetuses born from GBP+ women.

The specific parameter-matched comparative case study design was a methodological strength of our study. We intentionally matched the exact maternal age and the order of gravidity at the onset of GBP between cases and controls to limit the effects of age on metabolic disorders, and the effects of number of pregnancies on the alteration of immune response during pregnancy, respectively. The paucity of GBP cases in real practice precludes a study with large number of cases that weakens our conclusion. Future studies with larger numbers of GBP cases are needed to confirm our current findings. Moreover, we would like to suggest that as physiological and immunological changes occur among the subsequent orders of pregnancy apart from the age-dependent variations, both maternal age and the order of pregnancy need to be considered as matching parameters in future studies to strengthen the conclusions.

\section{Conclusion}

We found no association between GBP and PE as frequently mentioned in the previous case studies. Our findings of significantly higher SBP and DBP in the pregnant women with GBP may suggest the possibility of a shared pathogenesis of immune-mediated microvaculopathy causing GBP and PE. Further studies to explore the pathogenesis of GBP in relation to reactivation of latent HHV infections and alteration of maternal immune reaction are needed for elucidation.

\section{Declarations}

\section{Ethical considerations and consent to participate}

The study protocol was approved by the Ethics Committee of the Faculty of Medicine, Prince of Songkla University (EC Approval Registration No. 60-132-14-4) We strictly followed the regulations of the 1964 Declaration of Helsinki and its following amendments. We also followed current good clinical practice guidelines in doing this research. All patient information was completely anonymized. The consent to access the information of the patients in this study was granted under exemption condition by the institutional Ethics Committee of the Faculty of Medicine, Prince of Songkla University on behalf of all the participants in this study. 
Not applicable

\section{Availability of Data and Materials}

All the study data generated and analyzed during the current study are included in this published article.

\section{Competing interests}

All authors declare that they have no competing interests

\section{Funding and financial support}

No funding, financial or non-financial support related to this study and writing of the manuscript was received.

\section{Author contributions}

\section{S Leelawai M.D.}

Data acquisition, data analysis and interpretation, drafted the first version of the manuscript and approved the final manuscript.

C Suwanrath M.D. Interpretation of the analyzed data, assisted in revision and editing of the manuscript, and approved the final version before submission.

N Pruphetkaew M.Sc. data analysis and interpretation, and approved the final manuscript.

P Chongphattararot B.Sc. data analysis and interpretation, and approved the final manuscript.

P Sathirapanya* M.D. (Corresponding author) Conceptualization of the study, designed the study methods, interpretation of the analyzed data, revision and editing of the final manuscript, and approved the final manuscript before submission.

\section{Acknowledgements}

The authors would like to thank Dr. Thammasin Ingviya for his suggestions concerning the methodology, design and statistical analysis. We also thank David Patterson of the International Affairs Unit, Faculty of Medicine, Prince of Songkla University for editing the English in the manuscript.

\section{References}

1. Eviston TJ, Croxson GR, Kennedy PG, Hadlock T, Krishnan AV. Bell's palsy: aetiology, clinical features and multidisciplinary care. J Neurol Neurosurg Psychiatry. 2015;86:1356-61. doi: 10.1136/jnnp-2014309563. Epub 2015 Apr 9. PMID: 25857657. 
2. Jafri S, Ormiston ML. Immune regulation of systemic hypertension, pulmonary arterial hypertension, and preeclampsia: shared disease mechanisms and translational opportunities. Am J Physiol Regul Integr Comp Physiol. 2017;313:r693-r705.

3. Shmorgun D, Chan WS, Ray JG. Association between Bell's palsy in pregnancy and pre-eclampsia. QJM. 2002;95:359-62.

4. Katz A, Sergienko R, Dior U, Wiznitzer A, Kaplan D M, Sheiner E. Bell's palsy during pregnancy: is it associated with adverse perinatal outcome? Laryngoscope. 2011;121:1395-8.

5. Pourrat O, Neau JP, Pierre F. Bell's palsy in pregnancy: underlying HELLP syndrome or preeclampsia? Obstet Med. 2013;6:132-3. doi:10.1258/om.2012.110093.

6. Mylonas I, Kastner R, Sattler C, Kainer F, Friese K. Idiopathic facial paralysis (Bell's palsy) in the immediate puerperium in a patient with mild preeclampsia: a case report. Arch Gynecol Obstet. 2005;272:241-3.

7. Vrabec JT, Isaacson B, Van Hook JW. Bell's palsy and pregnancy. Otolaryngol Head Neck Surg. 2007;137:858-61. doi:10.1016/j.otohns.2007.09.009.

8. Hilsinger RL Jr, Adour KK, Doty HE. Idiopathic facial paralysis, pregnancy, and the menstrual cycle. Ann Otol Rhinol Laryngol. 1975;84(4 Pt 1):433-42. doi: 10.1177/000348947508400402. PMID: 168802.

9. Faas MM, De Vos P. Innate immune cells in the placental bed in healthy pregnancy and preeclampsia. Placenta. 2018;69:125-33.

10. LaMarca B, Cornelius DC, Harmon AC, et al. Identifying immune mechanisms mediating the hypertension during preeclampsia. Am J Physiol Regul Integr Comp Physiol. 2016;311:R1-R9. doi:10.1152/ajpregu.00052.2016.

11. Walters BN, Redman CW. Bell's palsy and cytomegalovirus mononucleosis in pregnancy. J R Soc Med. 1984;77:429-30. PMID: 6327983; PMCID: PMC1439928.

\section{Tables}

Table 1. Comparison of maternal characteristics and pregnancy-related metabolic disorders between the study pregnant women with and without gestational Bell's palsy by univariable analysis 
General characteristics

\begin{tabular}{|c|c|c|c|}
\hline Age, years, median (IQR) & $31.5(26.8,36.2)$ & $31.5(26.8,36.2)$ & 1 \\
\hline \multirow[t]{2}{*}{ Body weight, kg, median (IQR) } & $67(65.2,71.8)$ & & 0.353 \\
\hline & & $66.2(62.2,69.8)$ & \\
\hline Body height, cm, mean (SD) & $156.2(6.7)$ & $155.7(5.4)$ & 0.853 \\
\hline BMI mean, kg/m², mean (SD) & $29.8(5.2)$ & $27.3(2.9)$ & 0.091 \\
\hline GPA, n (\%) & & & 1 \\
\hline G1 & $3(37.5)$ & $15(37.5)$ & \\
\hline G2P1 & $3(37.5)$ & $15(37.5)$ & \\
\hline G4P3 & $1(12.5)$ & $5(12.5)$ & \\
\hline \multirow[t]{2}{*}{ G5P4 } & $1(12.5)$ & $5(12.5)$ & \\
\hline & & & 1 \\
\hline Association with GDM, GHT, & $144(95,147)$ & $130(116,146.5)$ & \\
\hline \multicolumn{4}{|l|}{ Preeclampsia and eclampsia } \\
\hline \multicolumn{4}{|l|}{ 1-hr 50-g GCT, mg/dL, median (IQR) } \\
\hline \multirow[t]{2}{*}{ SBP, mmHg, median (IQR) } & & & $0.045^{*}$ \\
\hline & $125(114.2,127.5)$ & $110(107,116)$ & \\
\hline \multicolumn{4}{|l|}{ DBP, mmHg, median (IQR) } \\
\hline & $77(73,80.8)$ & $70(65,73.2)$ & $0.021 *$ \\
\hline & & & 0.429 \\
\hline \multicolumn{4}{|l|}{ GDM, n, (\%) } \\
\hline Yes & $1(12.5)$ & $2(5)$ & \\
\hline No & 7 (87.5) & $38(95)$ & \\
\hline GHT, n, (\%) & & & 0.167 \\
\hline Yes & $1(12.5)$ & $0(0)$ & \\
\hline No & 7 (87.5) & $40(100)$ & \\
\hline Preeclampsia, n, (\%) & & & 0.167 \\
\hline
\end{tabular}




\begin{tabular}{|llll|} 
Yes & $1(12.5)$ & $0(0)$ & \\
No & $7(87.5)$ & $40(100)$ & 1 \\
\hline Eclampsia, n, (\%) & 0 & 0 & 1 \\
\hline
\end{tabular}

Abbreviations:

kg: kilogram(s), cm: centimeter(s), BMl: body mass index, GPA: gravida/para/abortus (GPA) system, 1hr 50-gram GCT: one-hour, 50-gram glucose challenge test, SBP: systolic blood pressure, DBP: diastolic blood pressure, mmHg: millimeters of mercury, GDM: gestational diabetes mellitus, GHT: gestational hypertension, SD: standard deviation, IQR: interquartile range

${ }^{*} \mathrm{p}<0.05$, Wilcoxon Rank Sum test

Table 2 Comparison of neonatal characteristics between newborns of the study pregnant women with and without gestational Bell's palsy by univariable analysis

\begin{tabular}{|llll|}
\hline Variable & Cases $(\mathbf{n = 9})$ & Controls $(\mathbf{n = 4 0 )}$ & p-value \\
\hline & $2672.2(744)$ & $3154.8(464.7)$ & $0.016^{*}$ \\
\hline Birth weight, kg, mean, (SD) & & & \\
\hline Birth length, cm, median, (IQR) & $49(48,54)$ & $50(49,51)$ & 0.927 \\
\hline Apgar 1, median, (IQR) & $9(8,9)$ & $9(9,9)$ & 0.438 \\
\hline Apgar 5, median, (IQR) & $10(9,10)$ & $10(9,10)$ & 0.917 \\
\hline Route of delivery & & & 0.064 \\
\hline Cesarean section & $7(77.8)$ & $16(40)$ & \\
\hline Normal labor & $2(22.2)$ & $24(60)$ & \\
\hline
\end{tabular}

$\mathrm{a}=$ one pair of twins, ${ }^{\star} p<0.05$

Abbreviations:

kg: kilogram(s), cm: centimeter(s), SD: standard deviation, IQR: interquartile range, Apgar 1: Apgar score at 1 minute after birth, Apgar 5: Apgar score at 5 minutes after birth. 